\title{
Force Density Relation and Lightweight Modeling of Single Layer Tensegrity Structures
}

Haoyu Yang1, Ruiwei Liu², Ani Luo, Heping Liu, Chuanyang Li

How to cite

Yang H (D) https: / / orcid.org/0000-0003-3626-1555

Liu R (D) https://orcid.org/ 0000-0002-5040-4594

Luo A (D) https: / / orcid.org/0000-0003-3644-4319

Liu H (iD https: / / orcid.org/0000-0002-0559-2311

Li C (DD https://orcid.org/0000-0003-0914-6746
Yang H; Liu R; Luo A; Liu H; Li C (2020) Force Density Relation and Lightweight Modeling of Single Layer Tensegrity Structures. J Aerosp Technol Manag, 12: e2620. https:// doi.org/10.5028/jatm.v12.1118

\begin{abstract}
A mathematical model is established using the space coordinates of nodes and vector matrix of components to study the construction method and lightweight nature of single-layer tensegrity structures on the basis of their geometric parameters. Connection matrix and configuration of the single-layer tensegrity structures are built using MATLAB software. The force balance equations of nodes of a three-bar tensegrity structure are established by introducing the force-density method, and the force-density relationship amongst the components is analysed. Thus, the configuration principle of single-layer tensegrity structure is verified. The force-density relationship between the components in the single-layer tensegrity structure is obtained. The change rule of the force-density relationship in different single-layer tensegrity structures is also analysed. Notably, $p$-1 stable configurations are present in the p-bar tensegrity structure. The force-density relationships of these p-1 configurations are in symmetrical distribution, that is, the j-th and (p-j)th configurations have the same force-density relationship. The lightweight nature of the structure is studied using the force-density relationship between the components, and the optimal structural parameter relationship is obtained when the structure has the lightest mass.
\end{abstract}

KEYWORDS: Tensegrity structure; Lightweight; Node vector matrix; Connection matrix; Force-density relationship.

\section{INTRODUCTION}

Tensegrity structure is a new type of spatial structure system and is composed of discrete bars and continuous cables. Figure 1 shows examples of common single-layer tensegrity structures.

Since the birth of tensegrity structure, scholars have carried out extensive research on it from different directions. Pellegrino and Calladine (1986) and Pellegrino (1990; 1993) proposed the classification of tensegrity structure. By using the self-stress mode number and displacement mode number of the structure, the geometric stability of the structure was determined by matrix analysis. The tangent stiffness matrix of the structure is obtained by the force balance equation and the physical meaning of each part of the tangent stiffness matrix is analysed (Guest 2006; 2011). On the basis of the tensegrity structure system classification theory proposed by Pellegrino and Calladine (1986) and Lazopulos et al. have made a more in-depth study on the determination of geometric stability of the IV class system (Lazopulos 2005a; b). Zhang et al. (2010) proposed a double-sided "star" tensegrity structure which on the basis of the tensegrity prism structure. Oliveira and Skelton $(2005 ; 2006)$ obtained the "tower" tensegrity structure by topological method, and completed the configuration and mechanical analysis of the structure (Masic and Skelton 2004). Luo et al. (2017) made a comprehensive and detailed study on the geometric stability and mobility of the tensegrity structure system (Luo 2000; Luo and Lu 2006). Luo et al. (2017) have systematically studied the theory of stable configuration of basic tensegrity units. 


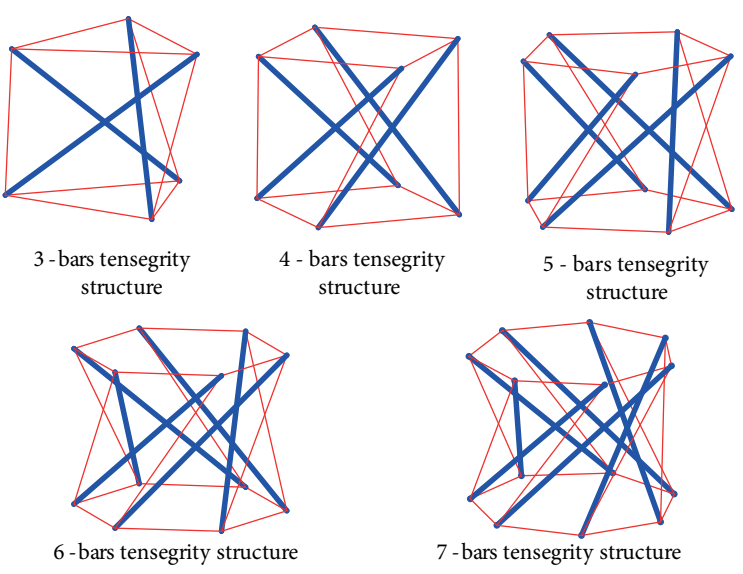

Figure 1. Common single-layer tensegrity structures.

At present, the research on the tensegrity structure is limited to the analysis of its mechanical properties or kinematics. No relevant research is available on realising lightweight of the structure and finding the optimal configuration. In this study, the mathematical and mechanical models of the structure are constructed on the basis of the generalised coordinates of the nodes of the basic unit, and the relationship between the configuration and the force-density of the structure is obtained. On the basis of the relationship of force-density between components, the parameter of material strength utilisation is introduced to measure the strength utilisation of components in the structure. A low-material strength utilisation indicates redundant dimensions and materials of the structure, a condition that requires light treatment. Therefore, the relationship between the geometric parameters of the single-layer tensegrity structure and the component parameters and the utilisation ratio of material strength was analysed and obtained. This method can be used to find the configuration with small mass under the same load condition. It can also guide the light treatment of the structure to ensure that its original strength can be guaranteed whilst the size and quality of the components are decreased. This method is applicable to all configurations of the single-layer tensegrity structure and can obtain the optimal configuration and structure size accurately and quickly.

\section{MATHEMATICAL MODEL OF SINGLE-LAYER TENSEGRITY}

The tensegrity structure is composed of nodes, cables and bars. The structural size of each component must be determined firstly prior to building a tensegrity structure. All the components are connected with the nodes. Thus, the mathematical model of the structure can be constructed from the node, and the relationship between the size of the component and the geometric parameters of the structure can be obtained. This study investigates a single-layer tensegrity structure of components with barycentres in one plane. The three-bar tensegrity structure is the most basic and simple element in the single-layer tensegrity structure. Thus, this study focuses mainly on the three-bar tensegrity structure. The force-density relationship between the members of the three-bar tensegrity structure is extended to other single-layer tensegrity structures.

Each single-layer tensegrity structure corresponds to a cylindrical envelop with a height $h$ and bottom radius $r$. If the number of bars is $p$, then the $p$-bar tensegrity structure consists of $2 p$ nodes and $3 p$ cables, and $p$ uniformly distributed nodes are present on the circumference of the upper and lower bottom of the enveloped cylinder. The nodes on the upper bottom surface may be connected to any nodes located on the lower bottom in addition to being on the same bar. Therefore, $p-1$ kinds of connecting modes of the inclined cables are present in the p-bar tensegrity structure, and $p-1$ different configurations can be constructed. As shown in Fig. 1, the centre of the lower bottom is the origin of the established Cartesian coordinate system. The angle of the $i$-th node on the lower bottom is

$$
\alpha_{d i}=\frac{2 \pi}{p} \cdot(i-1), \quad i \in[1, p]
$$


The coordinates of the nodes at the bottom are

$$
n_{d i}=\left[R \cos \alpha_{d i}, R \sin \alpha_{d i}, 0\right]^{T}, \quad i \in[1, p]
$$

The angle of the $i$-th node on the upper bottom is

$$
\alpha_{u i j}=\varphi_{j}+\frac{2 \pi}{p} \cdot(i-1)
$$

where $i \in[1, p], j \in[1, p-1], j$ is the configuration number and $\varphi_{j}$ is the torsion angle of the $j$-th configuration of the single-layer tensegrity structure (Oliveira and Skelton 2009), as shown in Fig. 2.

$$
\varphi_{j}=\frac{\pi}{2}+\frac{(p-j) \pi}{p}, j \in[1, p-1]
$$

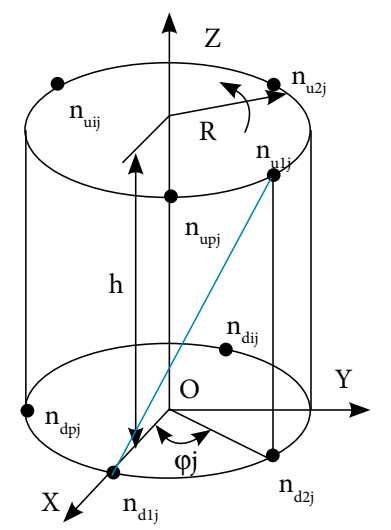

Figure 2. Node distribution graph.

The coordinates of the nodes at the upper surface are

$$
n_{u i j}=\left[R \cos \alpha_{u i j}, R \sin \alpha_{u i j}, h\right]^{T}, \quad i \in[1, p]
$$

The vector matrix $N_{j}$ of the node is

$$
N_{j}=\left[n_{d 1}, n_{d 2}, \cdots n_{d p}, n_{u 1 j}, n_{u 2 j}, \cdots n_{u p j}\right]_{3 \times 2 p}
$$

The component vector of the $i$-th bar can be expressed as

$$
\vec{b}_{i j}=n_{u i j}-n_{d i}, \quad i \in[1, p]
$$

The vectors of all bars are combined to form the bar vector matrix of the structure as follows:

$$
B_{j}=\left[\begin{array}{lll}
\vec{b}_{1 j} & \vec{b}_{2 j} \cdots & \vec{b}_{p j}
\end{array}\right]_{3 \times p}=N_{j} C_{B j}^{T}=N_{j}\left[\begin{array}{c}
-I \\
I
\end{array}\right]_{2 p \times p}
$$

where $C_{B j}^{T}$ is the bar connection matrix and matrix $I$ is a unit matrix of order $p$.

$$
C_{B j}^{T}=\left[\begin{array}{c}
-I \\
I
\end{array}\right]_{2 p \times p}
$$


The cable vector matrix can be represented as

$$
\begin{aligned}
& S_{j}=\left[\begin{array}{llllllllll}
\vec{S}_{h 1} & \cdots & \vec{S}_{h i} & \cdots & \vec{S}_{h 2 p} & \vec{S}_{v 1 j} & \cdots & \vec{S}_{v k j} & \cdots & \vec{S}_{v p j}
\end{array}\right]_{3 \times 3 p} \\
& =N_{j} C_{S j}^{T}=N_{j}\left[\begin{array}{ll}
C_{S h}^{T} & C_{S v j}^{T}
\end{array}\right]_{2 p \times 3 p}
\end{aligned}
$$

where $\vec{S}_{h i}$ is a horizontal cable, $i \in[1,2 p], \vec{S}_{v k j}$ is an inclined cable in the $\mathrm{j}$-th configuration and $k \in[1, p-1] . M^{2}$ atrix $N_{j}$ is the node coordinate matrix for the $\mathrm{j}$-th configuration. Matrix $C_{B j}^{T}$ is a cable connection matrix, which represents the connection relationship between node and cable vector matrices. $C_{S h}^{T}$ represents the connection matrix of the horizontal cable, and $C_{S v j}^{T}$ represents the connection matrix of the inclined cable.

$$
\begin{gathered}
C_{S h}^{T}=\left[\begin{array}{cc}
E & O \\
O & E
\end{array}\right]_{2 p \times 2 p} \\
E=\left[\begin{array}{cccccc}
-1 & 0 & 0 & \cdots & 0 & 1 \\
1 & -1 & 0 & \cdots & 0 & 0 \\
0 & 1 & -1 & \cdots & 0 & 0 \\
\vdots & 0 & 1 & \ddots & \vdots & \vdots \\
0 & 0 & 0 & \ddots & -1 & 0 \\
0 & 0 & 0 & \cdots & 1 & -1
\end{array}\right]_{p \times p}
\end{gathered}
$$

where

$$
\begin{aligned}
& C_{S v_{j}}^{T}=\left[\begin{array}{c}
-I \\
H
\end{array}\right]_{2 p \times p}, I \text { is a p-order unit matrix. } \\
& H=\left[\begin{array}{cc}
O & I_{j \times j} \\
I_{(p-j) \times(p-j)} & O
\end{array}\right]_{p \times p}
\end{aligned}
$$

where $I$ is the unit matrix, $j=12 \ldots p-1$.

The Solution of force-density relationship is as follows:

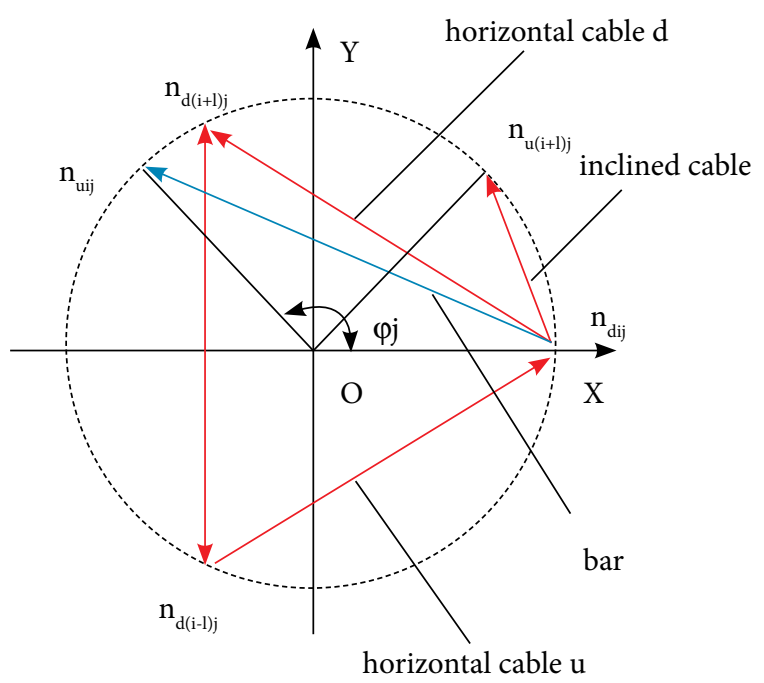

Figure 3. Top view of single layer tensegrity structure. 
The connection relationship of the node $n_{d i j}$ is shown in Fig. 3. In the figure, the thick arrow represents the bar and the thin arrow represents the cable. Each node is connected with two horizontal cables and one inclined cable. Given that the single-layer tensegrity structure is symmetrical, the stress of each node is the same. When the structure is in self-equilibrium state, each node is balanced by the internal force of the component. Force-density is introduced to determine the relationship between internal force and component vector ( $\mathrm{Lu} \mathrm{2008).} \mathrm{If} \mathrm{the} \mathrm{length} \mathrm{of} \mathrm{the} \mathrm{component} \mathrm{is} l$ and the internal force is $F$, then the force-density of the component is $F / l$. The forcedensity relationships of bar, inclined cable, horizontal cable $d$ and horizontal cable $u$ are represented by $\lambda, r_{v}, r_{h d}$ and $r_{h u}$, respectively.

For the nodes $n_{d i}$, the equations of force balance for the $x, y$ and $z$ axes are listed in Eq. $(11), i \in[1, p], j \in[1, p-1]$.

$$
\left[\begin{array}{ccc}
\left(x_{d i j}-x_{u i j}\right) & \left(x_{u(i+j) \mathrm{j}}-x_{d i j}\right)\left(x_{d(i+1) \mathrm{j}}-x_{d i j}\right) & \left(x_{d(i-1) \mathrm{j}}-x_{d i j}\right) \\
\left(y_{d i j}-y_{u i j}\right) & \left(y_{u(i+j) \mathrm{j}}-y_{d i j}\right)\left(y_{d(i+1) \mathrm{j}}-y_{d i j}\right) & \left(y_{d(i-1) \mathrm{j}}-y_{d i j}\right) \\
\left(z_{d i j}-z_{u i j}\right) & \left(z_{u(i+j) \mathrm{j}}-z_{d i j}\right)\left(z_{d(i+1) \mathrm{j}}-z_{d i j}\right) & \left(z_{d(i-1) \mathrm{j}}-z_{d i j}\right)
\end{array}\right]\left[\begin{array}{c}
\lambda \\
r_{v} \\
r_{h d} \\
r_{h u}
\end{array}\right]=0
$$

When $i+1>p, i+1=1$ is taken; when $i-1=0, i-1=p$ is taken. When the structure is subjected to external load $P$, the equilibrium equation is

$$
\left[\begin{array}{ccc}
\left(x_{d i j}-x_{u i j}\right) & \left(x_{u(i+j) \mathrm{j}}-x_{d i j}\right)\left(x_{d(i+1) \mathrm{j}}-x_{d i j}\right) & \left(x_{d(i-1) \mathrm{j}}-x_{d i j}\right) \\
\left(y_{d i j}-y_{u i j}\right) & \left(y_{u(i+j) \mathrm{j}}-y_{d i j}\right)\left(y_{d(i+1) \mathrm{j}}-y_{d i j}\right) & \left(y_{d(i-1) \mathrm{j}}-y_{d i j}\right) \\
\left(z_{d i j}-z_{u i j}\right) & \left(z_{u(i+j) \mathrm{j}}-z_{d i j}\right)\left(z_{d(i+1) \mathrm{j}}-z_{d i j}\right) & \left(z_{d(i-1) \mathrm{j}}-z_{d i j}\right)
\end{array}\right]\left[\begin{array}{c}
\lambda \\
r_{v} \\
r_{h d} \\
r_{h u}
\end{array}\right]=\left[\begin{array}{c}
P_{x} \\
P_{y} \\
P_{z}
\end{array}\right]
$$

The coefficient matrix is the equilibrium matrix $A_{d i j}$ of the single-layer tensegrity structure at the node $n_{d i j^{\circ}}$

$$
A_{d i j}=\left[\begin{array}{lll}
\left(x_{d i j}-x_{u i j}\right) & \left(x_{u(i+j) \mathrm{j}}-x_{d i j}\right)\left(x_{d(i+1) \mathrm{j}}-x_{d i j}\right) & \left(x_{d(i-1) \mathrm{j}}-x_{d i j}\right) \\
\left(y_{d i j}-y_{u i j}\right) & \left(y_{u(i+j) \mathrm{j}}-y_{d i j}\right)\left(y_{d(i+1) \mathrm{j}}-y_{d i j}\right) & \left(y_{d(i-1) \mathrm{j}}-y_{d i j}\right) \\
\left(z_{d i j}-z_{u i j}\right) & \left(z_{u(i+j) \mathrm{j}}-z_{d i j}\right)\left(z_{d(i+1) \mathrm{j}}-z_{d i j}\right) & \left(z_{d(i-1) \mathrm{j}}-z_{d i j}\right)
\end{array}\right]
$$

The coordinates of the bottom nodes and the upper bottom of the single-layer tensegrity structure are brought into Eq. 12 . Accordingly, the general formula of the equilibrium matrix $A$ of the single-layer tensegrity structure can be obtained as:

where

$$
A=\left[\begin{array}{llll}
a_{11} & a_{12} & a_{13} & a_{14} \\
a_{21} & a_{22} & a_{23} & a_{23} \\
a_{31} & a_{31} & a_{33} & a_{34}
\end{array}\right]
$$

$$
\begin{aligned}
& a_{11}=\left(R \cos \left(\frac{2 \pi(i-1)}{p}\right)-R \cos \left(\varphi_{j}+\frac{2 \pi(i+j-1)}{p}\right)\right. \\
& a_{12}=\left(R \cos \left(\varphi_{j}+\frac{2 \pi(i+j-1)}{p}\right)-R \cos \left(\frac{2 \pi(i-1)}{p}\right)\right) \\
& a_{13}=\left(R \cos \frac{2 \pi i}{p}-R \cos \left(\frac{2 \pi(i-1)}{p}\right)\right) \\
& a_{14}=\left(R \cos \frac{2 \pi(i-2)}{p}-R \cos \left(\frac{2 \pi(i-1)}{p}\right)\right) \\
& a_{21}=\left(R \sin \left(\frac{2 \pi(i-1)}{p}\right)-R \sin \left(\varphi_{j}+\frac{2 \pi(i+j-1)}{p}\right)\right. \\
& a_{22}=\left(R \sin \left(\left(\varphi_{j}+\frac{2 \pi(i+j-1)}{p}\right)\right)-R \sin \left(\frac{2 \pi(i-1)}{p}\right)\right) \\
& a_{23}=\left(R \sin \frac{2 \pi i}{p}-R \sin \left(\frac{2 \pi(i-1)}{p}\right)\right) \\
& a_{24}=\left(R \sin \frac{2 \pi(i-2)}{p}-R \sin \left(\frac{2 \pi(i-1)}{p}\right)\right) \\
& a_{31}=(0-h) \\
& a_{32}=(h-0) \\
& a_{33}=(0-0) \\
& a_{34}=(0-0)
\end{aligned}
$$


Equation 13 is a general form of the balance matrix $A$ of the single-layer tensegrity structure, where $j=12 \ldots p-1$. By substituting Eq. 13 into Eq. 10 and solving the matrix, the equation of force-density under no-load conditions can be obtained as:

$$
\left\{\begin{array}{l}
a_{11} \lambda+a_{12} r_{v}+a_{13} r_{h d}+a_{14} r_{h u}=0 \\
a_{21} \lambda+a_{22} r_{v}+a_{23} r_{h d}+a_{24} r_{h u}=0 \\
a_{31} \lambda+a_{32} r_{v}+a_{33} r_{h d}+a_{34} r_{h u}=0
\end{array}\right.
$$

Considering that the lengths and forces of the two horizontal cables connected to the same node are the same, we have:

$$
r_{h d}=r_{h u}
$$

By substituting Eq. 15 into Eq. 14, the right-hand side density relationship can be written as:

$$
\begin{aligned}
& r_{v}=\lambda \\
& r_{h d}=r_{h u}=\frac{\cos \left(\varphi_{j}+\frac{2 \pi(i-1)}{p}\right)-\cos \left(\varphi_{j}+\frac{2 \pi(i+j-1)}{p}\right)}{\cos \left(\frac{2 \pi i}{p}\right)+\cos \left(\frac{2 \pi(i-2)}{p}\right)-2 \cos \left(\frac{2 \pi(i-1)}{p}\right)} \lambda \\
& r_{h d}=r_{h u}=\frac{\sin \left(\varphi_{j}+\frac{2 \pi(i-1)}{p}\right)-\sin \left(\varphi_{j}+\frac{2 \pi(i+j-1)}{p}\right)}{\sin \left(\frac{2 \pi i}{p}\right)+\sin \left(\frac{2 \pi(i-2)}{p}\right)-2 \sin \left(\frac{2 \pi(i-1)}{p}\right)} \lambda
\end{aligned}
$$

The ultimate force-density relationship can be obtained by bringing $\varphi_{j}$ into simplification as follows:

$$
\begin{gathered}
r_{v}=\lambda \\
r_{h d}=r_{h u}=\frac{-\sin \left[\frac{(p-j) \pi}{p}+\frac{2 \pi(i-1)}{p}\right]+\sin \left[\frac{(p-j) \pi}{p}+\frac{2 \pi(i+j-1)}{p}\right]}{\cos \left(\frac{2 \pi i}{p}\right)+\cos \left[\frac{2 \pi(i-2)}{p}\right]-2 \cos \left[\frac{2 \pi(i-1)}{p}\right]} \lambda \\
r_{h d}=r_{h u}=\frac{\cos \left[\frac{(p-j) \pi}{p}+\frac{2 \pi(i-1)}{p}\right]-\cos \left[\frac{(p-j) \pi}{p}+\frac{2 \pi(i+j-1)}{p}\right]}{\sin \left(\frac{2 \pi i}{p}\right)+\sin \left[\frac{2 \pi(i-2)}{p}\right]-2 \sin \left[\frac{2 \pi(i-1)}{p}\right]} \lambda
\end{gathered}
$$

Equation 17 indicates that the force-density relationship of single-layer tensegrity structure is only related to the torsion angle and has nothing to do with the size of the member. The torsion angle is determined by the bar number $p$ and the configuration number $j$ of the structure. By satisfying Eq. 17, the structure can reach the self-equilibrium state.

The force-density of the inclined cable is always equal to that of the bar, and the force-density ratio of horizontal cable to bar varies with the numbers of bars and configurations. Eq. 17(b) and (c) represent the ratio of the force-density of the horizontal cable to the bar, and the reason for the two situations is that the nodes of the analysis are different. If the node is on the $x$ axis, then the denominator of Eq. 17 (c) is 0 , and Eq. 17(b) should be used. If the node is on the $y$ axis, then the denominator of Eq. 17(b) is 0, and Eq. 17(c) should be used. For other nodes, Eqs. 17(b) and (c) can be used for calculation to obtain the same results.

The stress of each node in the single-layer tensegrity structure is the same. Thus, $i=1$ was brought into Eq. 18 for ease of calculation: 


$$
\left\{\begin{array}{l}
r_{v}=\lambda \\
r_{h d}=r_{h u}=\frac{-\sin \left[\frac{(p-j) \pi}{p}\right]+\sin \left[\frac{(p+j) \pi}{p}\right]}{\cos \left(\frac{2 \pi}{p}\right)+\cos \left(\frac{2 \pi(p-1)}{p}\right)-2} \lambda
\end{array}\right.
$$

Equation 18 can obtain Eq. 19 by simplification to establish a simplified general formula of the force-density relationship of single-layer tensegrity structure:

$$
\left\{\begin{array}{l}
r_{v}=\lambda \\
r_{h d}=r_{h u}=\frac{\sin \left(\frac{j \pi}{p}\right)}{2 \sin ^{2}\left(\frac{\pi}{p}\right)} \lambda
\end{array}\right.
$$

Using the general formula of the force-density relationship of the single-layer tensegrity structure, we solve the force-density relationship of other common single-layer tensegrity structure. Figure 4 shows the relationship between the force-density ratio of members and the numbers of bars $p$ and configurations $j$.

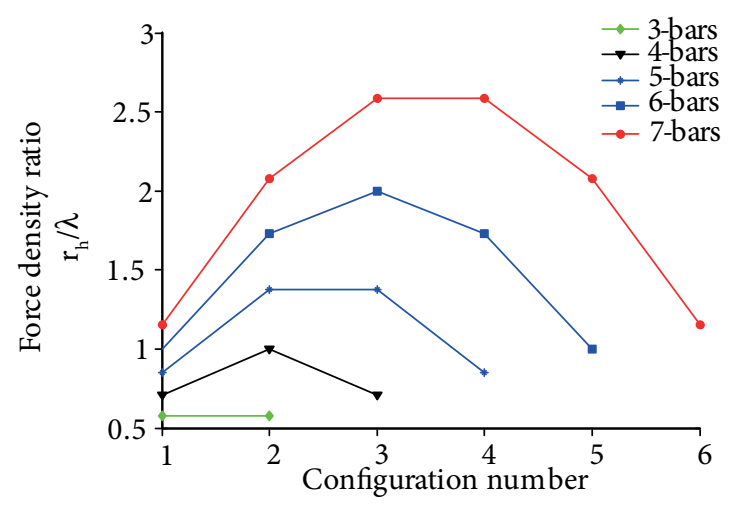

Figure 4. Relationship of force density of common single layer tensegrity structure.

The force-density of the declining cable is always the same as that of the bar. Thus, Fig. 4 shows only the ratio of force-density of the horizontal cable to the bar under different numbers of bars $p$ and configurations $j$. The ratio of force-density of the horizontal cable to the bar increases with the increase in the number of bars in the same kind of configuration (the configuration number $j$ is the same) of the single-layer tensegrity structure. Notably, $p$-1 kinds of different configurations of the $p$-bars tensegrity structure are considered, and the force-density relationship of all the configurations of each tensegrity structure is symmetrical.

\section{STRUCTURAL LIGHTWEIGHT MODELLING BASED ON FORCE-DENSITY RELATIONSHIP}

To achieve the minimum mass of the structure under the condition of certain bearing capacity and external load, the strength of the material must be utilised, the utilisation ratio of material strength improved and the stress distribution made as reasonable as possible. That is, the members with high strength have greater force, whereas those with less strength have lesser stress. The following conditions are required to ensure that the material is not damaged: 


$$
\frac{l \lambda}{A} \leq[\sigma]
$$

where $l$ is the length of the component, $A$ is the section area, $\lambda$ is the force-density and $[\sigma]$ is the allowable stress of the material.

To ensure the lightweight nature of the structure, the bar and cable should reach the allowable stress of the material at the same time as far as possible, that is,

$$
\frac{\frac{l_{b} \cdot \lambda_{b}}{A_{b}}}{\frac{l_{c} \cdot \lambda_{c}}{3 A_{c}}}=\frac{\left[\sigma_{b}\right]}{\left[\sigma_{c}\right]}
$$

Equation 21 is further processed into Eq. 22, which can be used to characterise the utilisation ratio of material strength of the structure. When the parameter $u$ is close to 0 , the utilisation of material strength is high.

$$
u=\sqrt{\left(\frac{3 l_{b} \lambda_{b} A_{c}}{A_{b} l_{c} \lambda_{c}} \cdot \frac{\left[\sigma_{c}\right]}{\left[\sigma_{b}\right]}-1\right)^{2}}
$$

The structure has three kinds of members: the bar, horizontal cable and inclined cable. Thus, Eq. 22 can be divided into two parts.

$$
u=\sqrt{\left(\frac{l_{b} \lambda_{b} A_{c h}}{A_{b} l_{c h} \lambda_{c h}} \cdot \frac{\left[\sigma_{c h}\right]}{\left[\sigma_{b}\right]}-1\right)^{2}}+\sqrt{\left(\frac{2 l_{b} \lambda_{b} A_{c v}}{A_{b} l_{c v} \lambda_{c v}} \cdot \frac{\left[\sigma_{c v}\right]}{\left[\sigma_{b}\right]}-1\right)^{2}}
$$

where $l_{b}$ is the length of the bar, $l_{c h}$ is the length of the horizontal cable and $l_{c v}$ is the length of the inclined cable.

The length of each component can be obtained from Eq. 2 and 5:

$$
\begin{aligned}
& l_{b}=\sqrt{\left(R \cos \varphi_{j}-R\right)^{2}+\left(R \sin \varphi_{j}\right)^{2}+h^{2}} \\
& l_{c h}=\sqrt{\left(R-R \cos \left(\frac{2 \pi}{p}\right)\right)^{2}+\left(R \sin \left(\frac{2 \pi}{p}\right)\right)^{2}} \\
& l_{c v}=\sqrt{\left(R-R \cos \varphi_{j}\right)^{2}+\left(R \sin \varphi_{j}\right)^{2}+h^{2}} \\
& \varphi_{j}=\frac{\pi}{2}+\frac{(p-j) \pi}{p}
\end{aligned}
$$

Equation 25 can be obtained by Eqs. 23 and 24:

$$
u=\sqrt{\left(\frac{\left[\sigma_{c h}\right] \sqrt{\left(\cos \varphi_{j}-1\right)^{2}+\sin ^{2} \varphi_{j}+\left(\frac{h}{R}\right)^{2}}}{\left(\frac{r_{h}}{\lambda}\right)\left(\frac{h}{R}\right)^{2}\left[\sigma_{b}\right] \sqrt{\left(1-\cos \left(\frac{2 \pi}{p}\right)\right)^{2}+\sin ^{2}\left(\frac{2 \pi}{p}\right)}}-1\right)^{2}}+\sqrt{\left(\frac{2\left[\sigma_{c v}\right] \sqrt{\left(\cos \varphi_{j}-1\right)^{2}+\sin ^{2} \varphi_{j}+\left(\frac{h}{R}\right)^{2}}}{\left(\frac{r_{b}}{r_{c}}\right)^{2}\left(\frac{r_{v}}{\lambda}\right)\left[\sigma_{b}\right] \sqrt{\left(1-\cos \varphi_{j}\right)^{2}+\sin ^{2} \varphi_{j}+\left(\frac{h}{R}\right)^{2}}}-1\right)^{2}}
$$

Equation 25 indicates that the utilisation ratio of material strength is related to the configuration number $j$ of structure, radius ratio of bar to cable $r_{b} / r_{c}$ and length-diameter ratio of enveloped cylinder of structure $h / R$. The influence of each parameter on the utilisation ratio of material strength of a three-bar tensegrity structure is analysed by the method of controlling variables. 


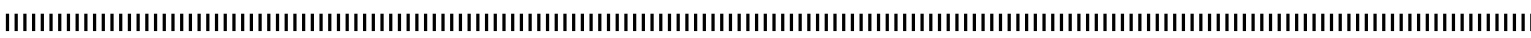

Figure 4 shows the effect of the radius ratio of bar to cable $r_{b} / r_{c}$ and the ratio of enveloped cylinder of structure $h / R$ on the parameter $u$ after the material of the bar and cable is determined. In the figure, the $z$ axis represents the parameter $u$, the $x$ axis represents the radius ratio of the bar to the cable $r_{b} / r_{c}, y$ axis represents the length-diameter ratio of enveloped cylinder of structure $h / R$ and $m_{31}$ is the valley line of the function. The point on the valley line represents the lowest $u$ value the structure can reach after a parameter of structure is determined. In other words, the points on the valley line are the best combination of structural parameters under the condition of realising lightweight.

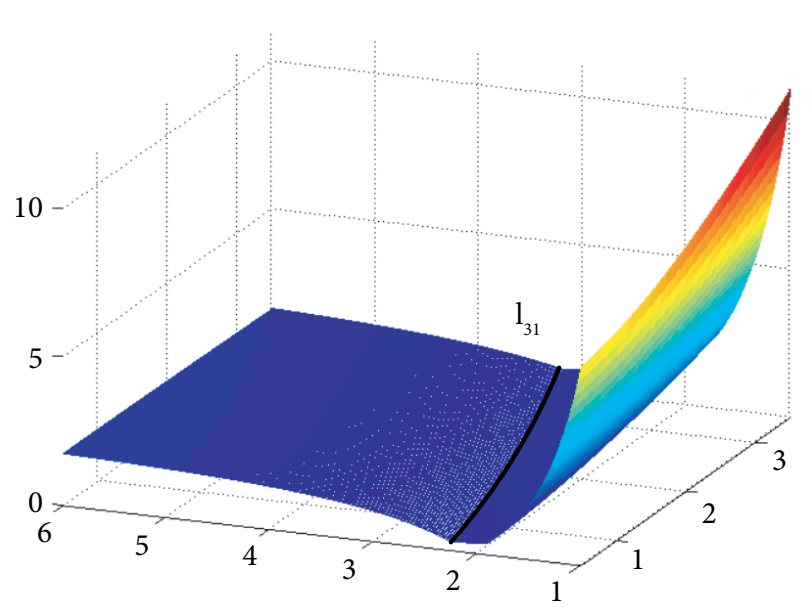

Figure 5. The relationship between parameters $u$ and $r_{\mathrm{b}} / r_{\mathrm{c}}$ and $h / R$.

Figure 5 shows that simply reducing or increasing $r_{b} / r_{c}$ and $h / R$ cannot ensure the high utilisation ratio of material strength of the structure and its lightweight nature. However, when both reach a specific value, the parameter $u$ can reach a low value, and the lightweight nature of the structure is realised. When one parameter is determined, the other parameter is also uniquely determined to guarantee the lightest mass of the structure. The projection of the valley line in Fig. 5 on the $x O y$ plane is the set of the best combinations of parameters $r_{b} / r_{c}$ and $h / R$, that is, the parameter combination on the valley value line can be a structure with the utilisation ratio of material strength.

By using the same method, other single-layer tensegrity structures and their configurations are analysed. The valley line $l_{p i}$ distribution obtained is shown in Fig. 6 . In the figure, $p$ is the number of bars and $j$ is the number of configurations.

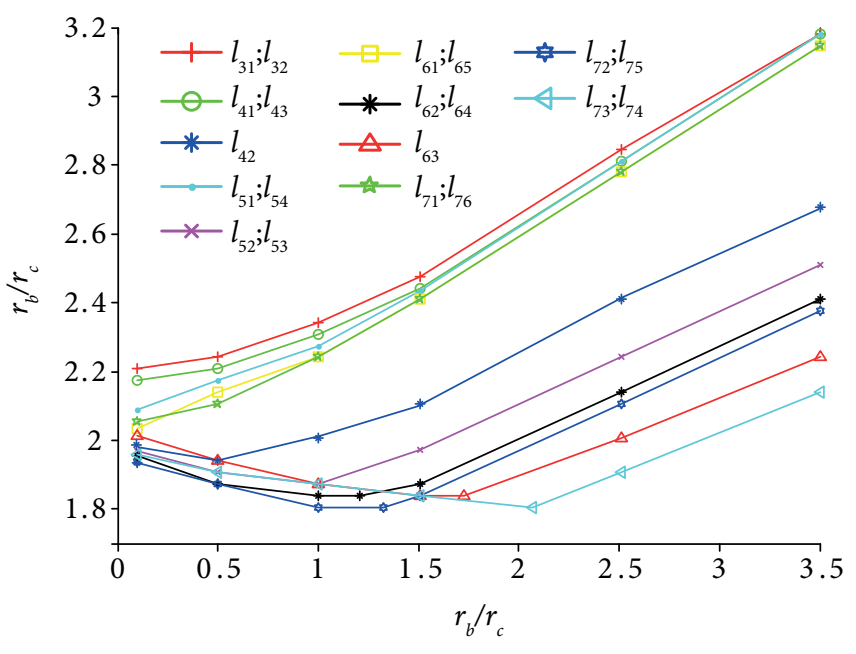

Figure 6. Peak line $l_{\mathrm{pj}}$ distribution. 
Figure 6 shows that, for the first and p-th configurations of each tensegrity structure, the radius ratio of bar to cable $r_{b} / r_{c}$ and the length-diameter ratio of enveloped cylinder of structure $h / R$ must be improved to achieve the optimum utilisation ratio of material strength as the number of bars increases. For other configurations, the projection of the valley line on the $x \mathrm{O} y$ plane has extreme points. Before the extreme point, a small force-density indicates that a small radius ratio of bar to cable $r_{b} / r_{c}$ and a small length-diameter ratio of enveloped cylinder of structure $h / R$ are needed. After the extreme point, a great force-density ratio indicates that a small radius ratio of bar to cable $r_{b} / r_{c}$ and a small length-diameter ratio of enveloped cylinder of structure $h / R$ are needed.

Figure 7 shows the combination of structural parameters when each configuration is at the highest utilisation ratio of material strength (parameter $u$ is at a minimum).

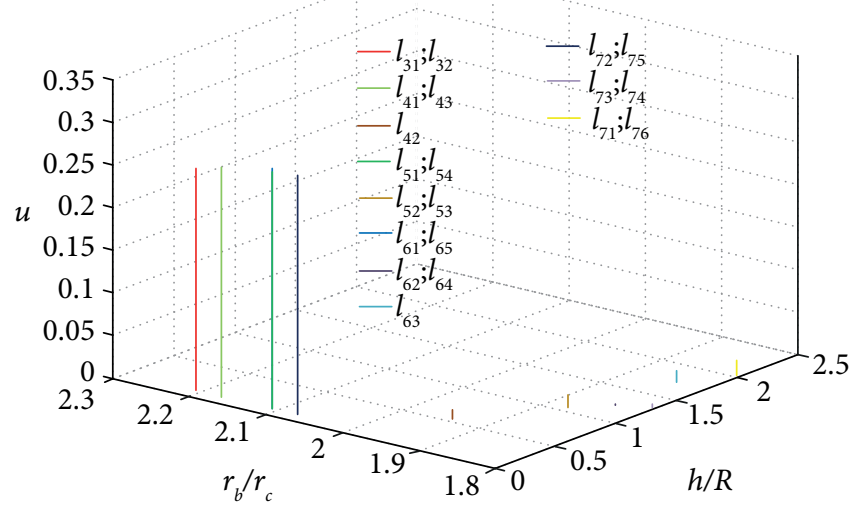

Figure 7. The optimal structural parameter combination for each configuration.

From Fig. 7, it can be found that the configurations with the same force-density ratio have the same utilisation ratio of material strength. When the ratio of force-density is large, the utilisation ratio of material strength is high. For the first and ( $p$-1)th configurations of each tensegrity structure, the parameter $u$ has no extreme value in the interval, that is, a small length-diameter ratio of enveloped cylinder indicates a high utilisation ratio of material strength. Therefore, the optimal combination of structural parameters cannot be obtained.

Compared with the two configurations above, the utilisation ratio of material strength of other configurations is greatly improved, and an extreme value exists in the interval. Accordingly, the optimal combination of structural parameters can be obtained.

The optimal structural parameter combinations for each configuration in the interval are shown in Table 1 , in which $t_{p j}$ denotes the $j$-th configuration of $p$-bar tensegrity structure.

Table 1. Optimum structural parameters combination of each configuration.

\begin{tabular}{|c|c|c|c|c|c|}
\hline configuration & $\boldsymbol{h} / \boldsymbol{R}$ & $\boldsymbol{r}_{b} / \boldsymbol{r}_{c}$ & configuration & $\boldsymbol{h} / \boldsymbol{R}$ & $\boldsymbol{r}_{b} / \boldsymbol{r}_{c}$ \\
\hline$t_{31} ; t_{32}$ & 0.1 & 2.208 & $t_{62} ; t_{64}$ & 1.241 & 1.839 \\
\hline$t_{41} ; t_{43}$ & 0.1 & 2.174 & $t_{63}$ & 1.743 & 1.839 \\
\hline$t_{42}$ & 0.5336 & 1.94 & $t_{71} ; t_{76}$ & 0.1 & 2.074 \\
\hline$t_{51} ; t_{54}$ & 0.1 & 2.107 & $t_{72} ; t_{75}$ & 1.332 & 1.805 \\
\hline$t_{52} ; t_{53}$ & 1.058 & 1.872 & $t_{73} ; t_{74}$ & 2.206 & 1.805 \\
\hline$t_{61} ; t_{65}$ & 0.1 & 2.107 & & & \\
\hline
\end{tabular}

Figure 8 shows the relationship between the utilisation ratio of material strength and the force-density ratio for each configuration. The utilisation ratio of material strength is related to not only the force-density ratio but also the configuration of the structure. Different configurations have dissimilar distribution conditions of the bars and cables. The utilisation ratio of material strength of the second and ( $p$-2)th configurations of each tensegrity structure is high, which indicates that the distribution of bars and cables is reasonable. 


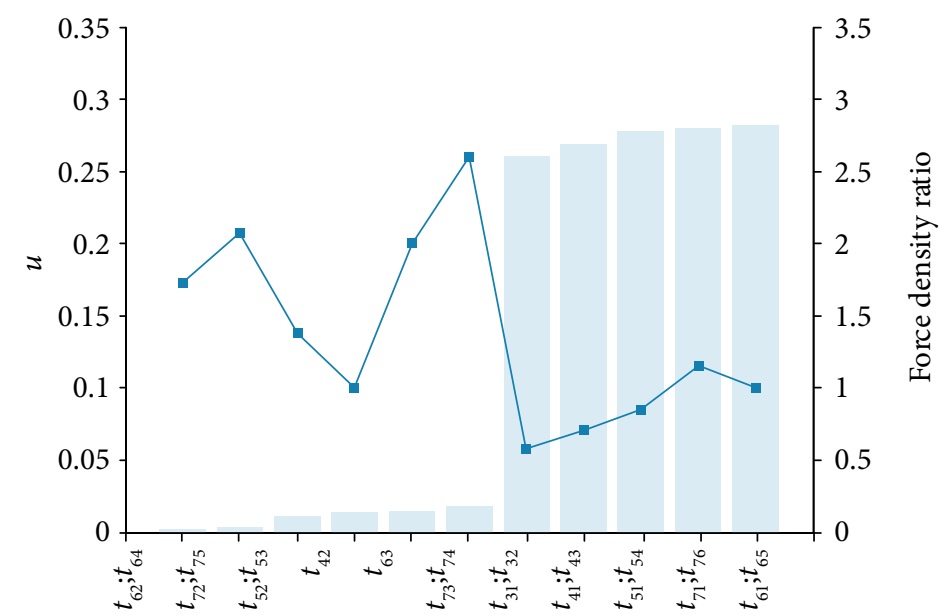

Figure 8. The relationship between utilisation ratio of material strength and force density ratio of each configuration.

The parameter $u$ is also related to the ratio of material strength of bars to cables. Figure 9 shows the relationship between parameter $u$ and ratio of material strength of bars to cables when the radius ratio of bar to cable and length-diameter ratio of enveloped cylinder of structure are determined.

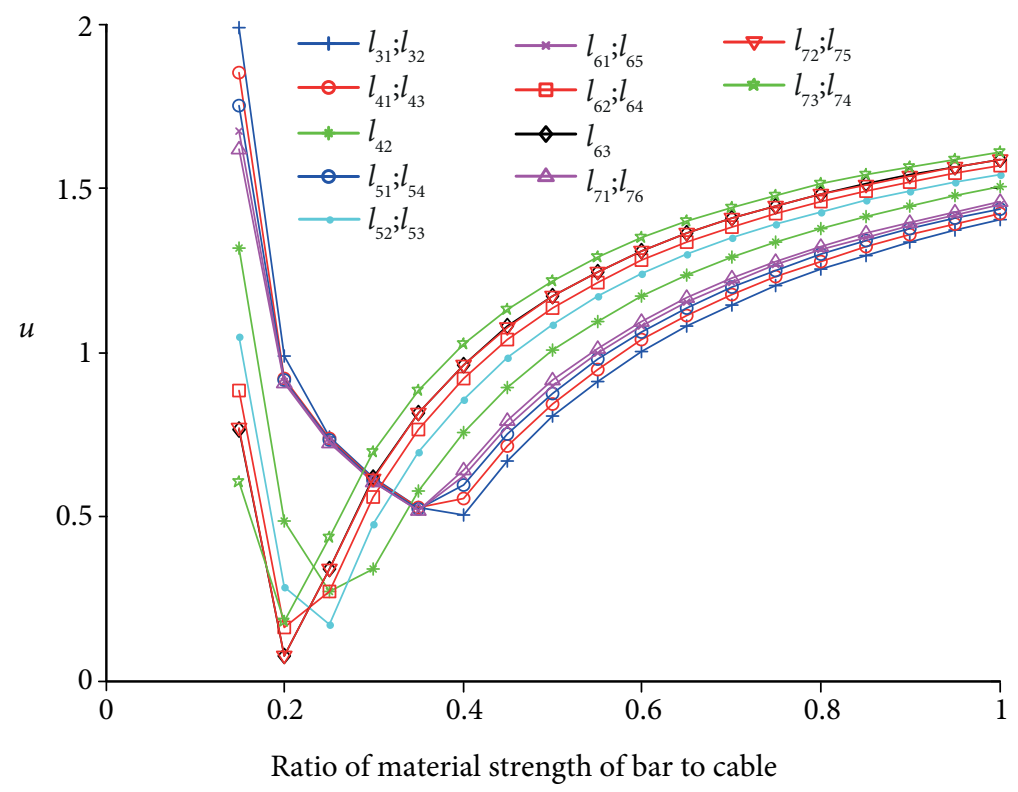

Figure 9. The relationship between parameter $u$ and the ratio of material strength of bar to cable.

In Eq. 22, the parameter $u$ is used to represent the utilisation ratio of material strength. When the parameter $u$ is close to 0 , the utilisation of material strength is high. Figure 9 shows that, for the first and ( $p$-1)th configurations of each tensegrity structure, the parameter $u$ is large in the interval. When the ratio of material strength of bars to cables is 0.4 , the utilisation ratio of material strength of the structure reaches the highest. Compared with the two configurations above, the utilisation ratio of material strength of other configurations is greatly improved. When the ratio of material strength of bars to cables is 0.2 , the utilisation ratio of material strength of the structure reaches the highest. 


\section{APPLICATION EXAMPLE}

Tibert (2002; Tibert and Pellegrino 2002; 2003; 2011) carried out structural model finding analysis and parametric modelling for the fifth configuration of the six-bar tensegrity structure, established mechanical model through force-density analysis and developed a tensegrity antenna based on the six-bar tensegrity structure in 2002 (Fig. 10). Stiffness analysis and extension test are also carried out. The extension process is shown in Fig. 11, which verifies that the single-layer tensegrity structure can be applied to the satellite antenna. The combined parameters of the model established by the authors are shown in Table 2. However, Tibert et al. (2002; Tibert and Pellegrino 2002; 2003; 2011) did not study how to realise the lightweight nature of the structure. Thus, this work used the analysis method described above to optimise the six-bar tensegrity structure for realising the lightweight nature of the structure.

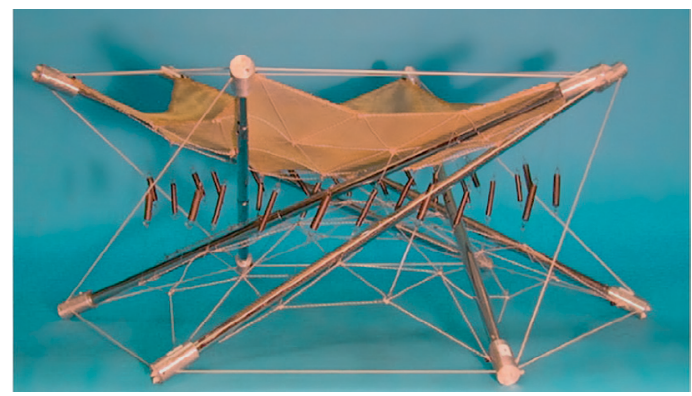

Figure 10. The tensegrity antenna.
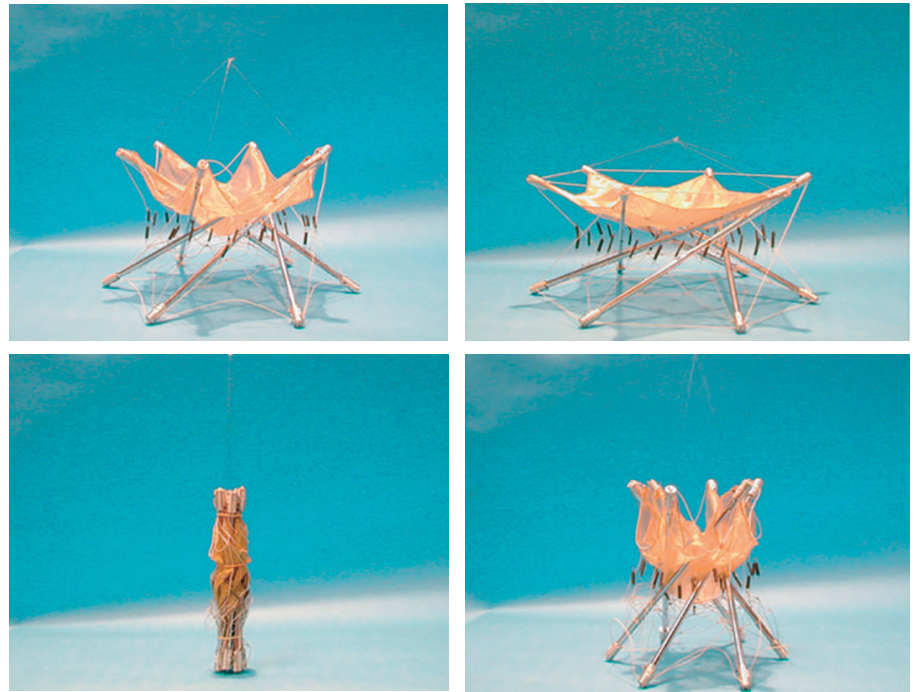

Figure 11. The development of the antenna.

Table 2. Antenna structure parameters.

\section{Bar number}

Bar diameter

Cable diameter

The height of the envelope cylinder

The diameter of the envelope cylinder

\section{6}

$0.01 \mathrm{~m}$

$0.01 \mathrm{~m}$

$0.2 \mathrm{~m}$

$0.45 \mathrm{~m}$ 
The cable in the structure is made of steel wire rope and the bar member is made of aluminium alloy. The geometric and material parameters of the structure are substituted into Eq. 25, and the parameters of the (Tibert, 2002; Tibert and Pellegrino 2002; 2003; 2011) model can be calculated as $u=3.32$. The parameter $u$ is large and the material strength utilisation ratio is low, thereby leading to the heavy mass of the structure. The geometric and material parameters of the structure are then substituted into Eq. 26 to obtain the mass of the model $\mathrm{m}=0.544 \mathrm{~kg}$ :

$$
m=\pi p l_{b} r_{b}^{2} \rho_{b}+\pi p l_{v} r_{v}^{2} \rho_{v}+2 \pi p l_{h} r_{h}^{2} \rho_{h}
$$

where $\rho_{b}, \rho_{v}$ and $\rho_{h}$ are the densities of the bar, diagonal cable and horizontal cable.

To improve the utilisation ratio of the structure material strength, the length-diameter ratio of enveloped cylinder of structure $h / R$ and the radius ratio of bar to cable $r_{b} / r_{c}$ should be reasonably adjusted. If the geometric shape of the structure should be kept unchanged, then only the radius ratio of bar to cable $r_{b} / r_{c}$ can be adjusted. The relationship between the radius ratio of bar to cable $r_{b} / r_{c}$ and the parameter $u$ is shown in Fig. 12. When $r_{b} / r_{c}=1.78$ and $\mathrm{u}=2.37$, the utilisation ratio of material strength is high. At this point, the structure mass is $\mathrm{m}=0.056 \mathrm{~kg}$. Thus, adjusting the radius ratio of bar to cable to reduce the parameter $u$ and increasing the utilisation ratio of material strength can decrease the structure quality considerably under the condition of guaranteed load bearing.

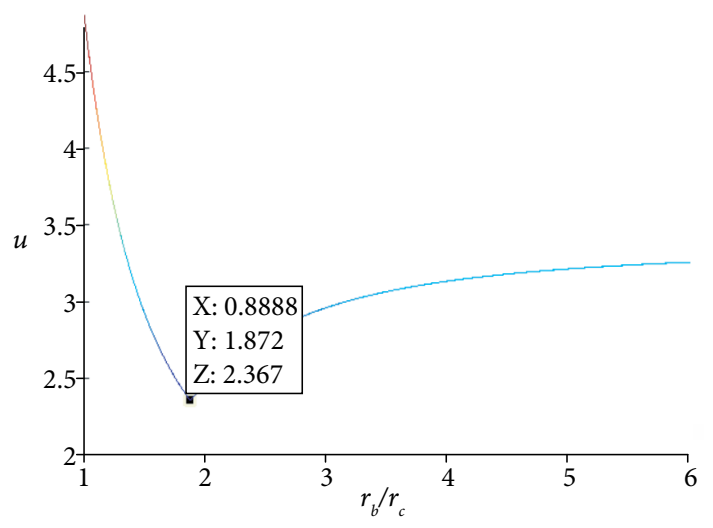

Figure 12. The relationship between the radius ratio of bar to cable $r_{b} / r_{c}$ and the parameter $u$.

As shown in Fig. 13, the six-bar tensegrity structure has five different configurations. The antenna developed by (Tibert, 2002; Tibert and Pellegrino 2002; 2003; 2011) uses the fifth configuration. However, this configuration does not necessarily have the minimum mass of the structure. Thus, other configurations need to be analysed. All the inclined cables in the first configuration meet at one point, and all the bars in the fourth configuration meet at one point. Therefore, they cannot be applied to the antenna. Only the second, third and fifth configurations need to be analysed.

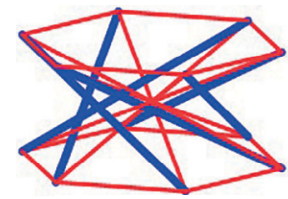

$p=6, j=1$

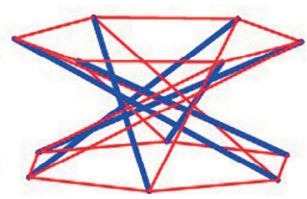

$p=6, j=2$

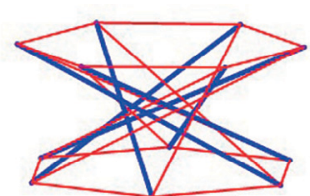

$p=6, j=3$

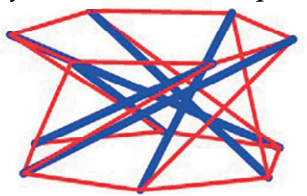

$p=6, j=4$

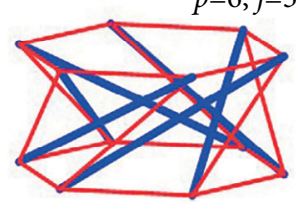

$p=6, j=5$

Figure 13. Five configurations of the six-bars tensegrity structure. 
The relationship between the parameters of the second configuration $u$ and the radius ratio of bar to cable $r_{b} / r_{c}$ is shown in Fig. 14 . When $r_{b} / r_{c}=1.74$ and $\mathrm{u}=0.90$, the utilisation ratio of material strength is improved, and the structure mass at this time is $\mathrm{m}=0.052 \mathrm{~kg}$. The relationship between the parameters of the third configuration $u$ and the radius ratio of bar to cable $r_{b} / r_{c}$ is shown in Fig. 15 . When $r_{b} / r_{c}=1.77$ and $\mathrm{u}=0.90$, the utilisation ratio of material strength is improved, and the structure mass at this time is $\mathrm{m}=0.053 \mathrm{~kg}$.

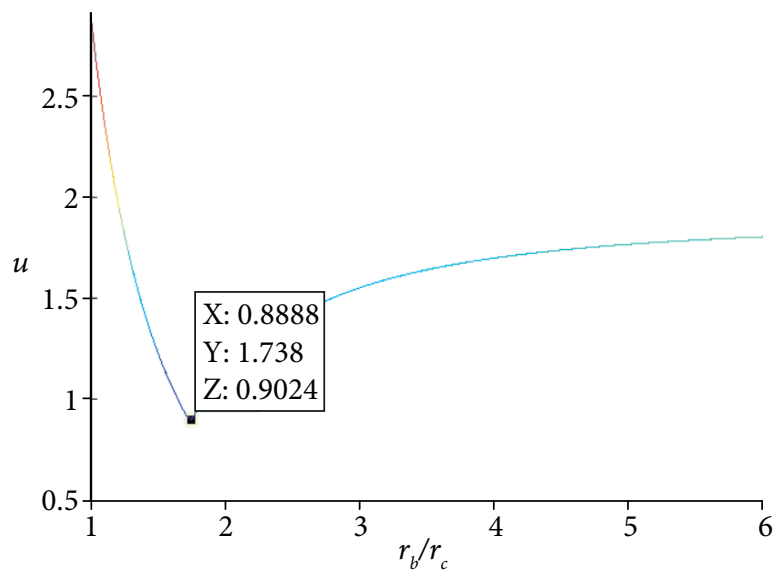

Figure 14. The relation between the parameters $u$ and $r_{b} / r_{c}$ in 2nd configuration.

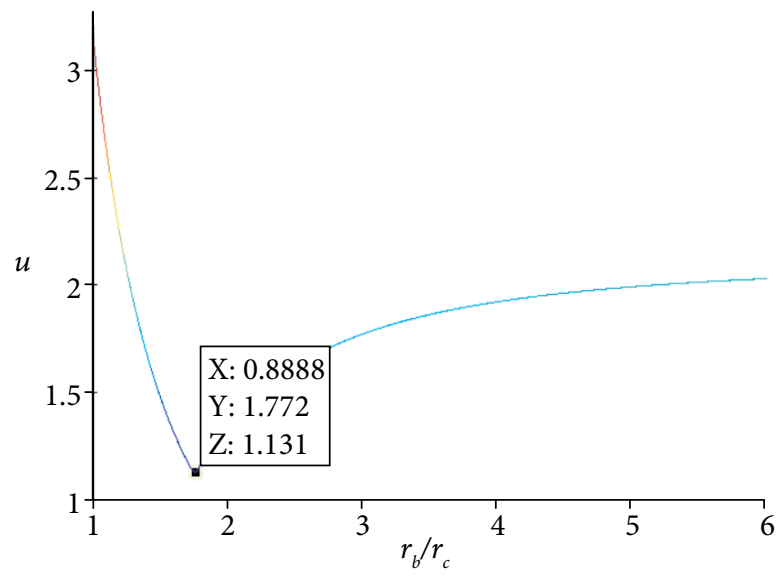

Figure 15. The relation between the parameters $u$ and $r_{b} / r_{c}$ in 3rd configuration.

The results of the three configurations are shown in Table 3. The parameter $u$ of the second configuration is the smallest, the material strength utilisation ratio is the highest and the mass is the smallest. The third configuration comes second in terms of mass, and the fifth configuration is the largest. However, the difference between the three configurations is inconsiderable. Therefore, the structure configuration only slightly influences the utilisation ratio of material strength after the radius and length-diameter ratios of the structure are determined.

Table 3. The results of the three configurations.

\begin{tabular}{|c|c|c|c|}
\hline Configuration & Parameter $u$ & $r_{b} / r_{c}$ & Quality $\mathrm{m} / \mathrm{kg}$ \\
\hline $\mathrm{j}=2$ & 0.90 & 1.74 & 0.052 \\
\hline $\mathrm{j}=3$ & 1.13 & 1.77 & 0.053 \\
\hline $\mathrm{J}=5$ & 2.37 & 1.87 & 0.056 \\
\hline
\end{tabular}


Figure 16 shows the relationship between the parameter $u$ and the structural length-diameter ratio in the second configuration when $r_{b} / r_{c}=1.74$. When the diameter $R$ of the antenna remains unchanged and the height $h$ of the antenna is decreased, the parameter $u$ increases and the utilisation ratio of material strength decreases. However, when the height of the antenna increases, the collection volume of the antenna increases. Therefore, the aspect ratio of the antenna also needs to be determined depending on the size of the rocket payload bay.

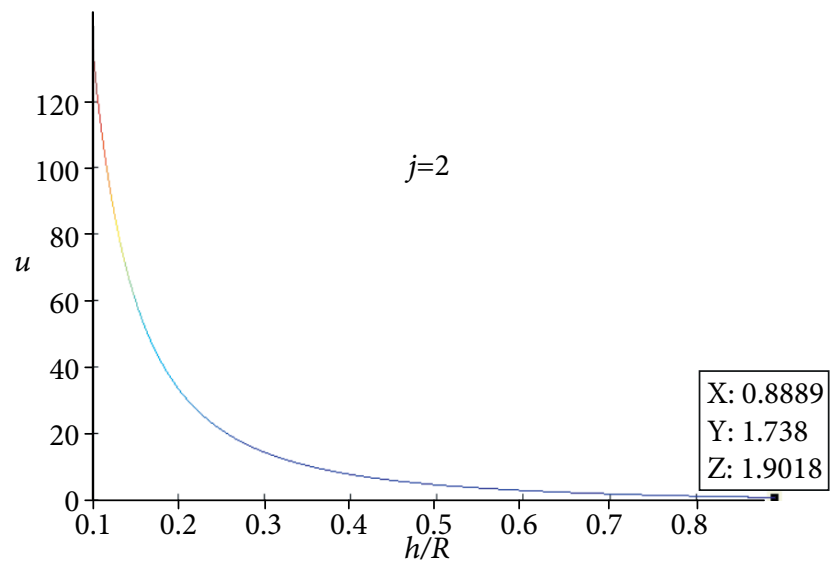

Figure 16. The relationship between the parameter $u$ and $h / R$ in the 2nd configuration.

\section{CONCLUSIONS}

On the basis of the geometric parameters of a three-bar tensegrity structure, the node generalised coordinate, component vector matrix and connection matrix are constructed. The mathematical model of a single-layer tensegrity structure based on node generalised coordinate is established. The force of the node through the force balance equation of the structure is analysed, and the concept of force-density is introduced. The internal force of the member is directly related to the component vector. On the basis of the node coordinate and connection matrix, the force balance matrix of the single-layer tensegrity structure and the force-density relationship between components are deduced, and the relationship between the forcedensity and the numbers of bars $p$ and configurations $j$ of the single-layer tensegrity structure is obtained. The concept of utilisation ratio of material strength is put forward, and the improvement in utilisation ratio of material strength is studied using the force-density relationship of components. The optimum structural parameter combination is obtained, and the aim of lightweight is achieved.

\section{FUNDING}

National Natural Science Foundation of China

Grant ns. 51575119, 51675114, U1613201, U1637207.

[http://dx.doi.org/10.13039/501100001809]

\section{ACKNOWLEDGMENTS}

Editors and authors are thankful to Fundação Conrado Wessel for providing the financial support for publishing this article. 


\section{AUTHOR'S CONTRIBUTION}

Conceptualization, Haoyu Yang and Ruiwei Liu; Methodology, Haoyu Yang; Investigation, Haoyu Yang; Writing - Original Draft, Haoyu Yang Ruiwei Liu and Chuanyang Li; Writing - Review \& Editing, Haoyu Yang; Funding Acquisition, Ani Luo and Heping Liu; Resources, Ani Luo and Heping Liu; Supervision, Haoyu Yang.

\section{REFERENCES}

Guest SD (2006) The stiffness of prestressed frameworks: A unifying approach. Int J Solids Struct. 43(3-4):842-854. https://doi. org/10.1016/j.ijsolstr.2005.03.008

Guest SD (2011) The stiffness of tensegrity structures. IMA J Appl Math. 76(1):57-66. https://doi.org/10.1093/imamat/hxqO65

Lazopulos KA (2005a) Stability of an elastic cytoskeletal tensegrity model. Int J Solids Struct. 42(11-12):3459-3469. https://doi. org/10.1016/j.ijsolstr.2004.11.008

Lazopulos KA (2005b) Stability of an elastic tensegrity structure. Acta Mech. 179(1-2):1-10. https://doi.org/10.1007/s00707-0050244-0

Lu J (2008) Research on equilibrium matrix analysis theory of kinematically indeterminate structures (Doctoral Thesis). Hangzhou: Zhejiang University.

Luo A, Liu H, Skelton RE, Che S (2017) The theory of basic tensegrity unit stable forming. Chinese J Mech Eng. 53(23):62-73. https:// doi.org/10.3901/JME.2017.23.062

Luo YZ (2000) Geometrical stability analysis of cable-strut tensile structures. J Zhejiang Univ-Sc. 27(6):608-611.

Luo YZ, Lu JY (2006) New criteria for the mobility of 3-D bar structure. J Eng Mech. 11:70-75.

Masic M, Skelton RE (2004) Optimization of class-2 tensegrity towers. In: Proceedings of the Society of Photo-Optical Instrumentation Engineers (SPIE). San Diego: SPIE. https://doi.org/10.1117/12.540363

Oliveira MC, Skelton RE. (2005) A new topology of tensegrity towers with uniform force distribution. In: Proceedings of the Society of Photo-Optical Instrumentation Engineers (SPIE). San Diego: SPIE. https://doi.org/10.1117/12.598265

Oliveira MC, Skelton RE (2009) Tensegrity systems. New York: Springer. https://doi.org/10.1007/978-0-387-74242-7

Oliveira MC, Skelton RE, Chan WL (2006) Minimum Mass Design of Tensegrity Towers and Plates. In: IEEE Conference on Decision and Control. New York: IEEE. https://doi.org/10.1109/CDC.2006.377427

Pellegrino S (1990) Analysis of prestressed mechanisms. Int J Solids Struct. 26(12):1329-1350. https://doi.org/10.1016/00207683(90)90082-7

Pellegrino S (1993) Structural computations with the singular value decomposition of the equilibrium matrix. Int J Solids Struct. 30(21):3025-3035. https://doi.org/10.1016/0020-7683(93)90210-X

Pellegrino S, Calladine CR (1986) Matrix analysis of statically and kinematically indeterminate frameworks. Int J Solids Struct. 22(4):409428. https://doi.org/10.1016/0020-7683(86)90014-4

Tibert AG (2002) Deployment tensegrity structure for space applications (Doctoral Thesis) Stockholm: Royal Institute of Technology, Department of Mechanics.

Tibert AG, Pellegrino S (2002) Deployable tensegrity reflectors for small satellites. J Space Eng. 39(5):707-709. https://doi. org/10.2514/2.3867

Tibert AG, Pellegrino S (2003) Deployable tensegrity masts. In: 44th AIAA/ASME/ASCE/AHS/ASC Structures, Structural Dynamics, and Materials Conference. Norfolk: Meeting Paper. https://doi.org/10.2514/6.2003-1978

Tibert AG, Pellegrino S (2011) Review of form-finding methods for tensegrity structures. Int J Space Struct. 18(4):209-223. https:// doi.org/10.1260/026635103322987940

Zhang JY, Guest DS, Connelly R, Ohsaki M (2010) Dihedral 'star' tensegrity structures. Int J Solids Struct. 47(1):1-9. https://doi. org/10.1016/j.ijsolstr.2009.05.018 